\title{
Transcatheter arterial chemoembolization improves the resectability of malignant breast phyllodes tumor with angiosarcoma component: a case report
}

\author{
Chih-Yu Kuo ${ }^{1+}$, Shing-Huey Lin ${ }^{2+}$, Kuan-Der Lee ${ }^{6}$, Sho-Jen Cheng ${ }^{7}$, Jan-Show Chu ${ }^{8}$ and Shih-Hsin Tu $3 u^{3,4,5^{*}}$
}

\begin{abstract}
Background: A giant phyllodes tumor of the breast is a rare fibroepithelial lesion, and its treatment is controversial. Many case reports have reported performing skin graft reconstruction after tumor excision. Chest wall resection may be required if the tumor has invaded the chest muscle layer. We speculated that transcatheter arterial chemoembolization (TACE) can improve the resectability of malignant phyllodes tumor of the breast without requiring skin grafting. The English literature contains only one case report similar to our experience.

Case presentation: We report a rare case of a 51-year-old woman who had a giant malignant phyllodes tumor with heterologous sarcomatous differentiation in her right breast. The tumor was $19.43 \times 12.98 \times 21.47 \mathrm{~cm}$. Wholebody computed tomography (CT) and bone scan did not reveal distant metastasis. Chest magnetic resonance imaging showed chest wall tumor invasion. Considering that skin defects after mastectomy can be extensive, we administered four courses of chemoembolization in the 5 weeks before surgery $(30 \mathrm{mg}$ of epirubicin and embozene microspheres $[400,500$, and $700 \mu \mathrm{m}] /$ week). Each process was well tolerated, with no serious complications. Only fever and local pain at the tumor site were noted, and these symptoms resolved with time. The follow-up CT scan showed a $45 \%$ reduction in tumor volume. Therefore, simple mastectomy was performed without skin grafting reconstruction. Wound healing was satisfactory, and the patient was discharged 1 week after surgery. Pathological and immunohistochemistry $(\mathrm{IHC})$ findings showed a malignant phyllodes tumor with an angiosarcoma component. Because of tumor invasion of the chest wall, we recommended the patient receive radiotherapy, but she refused. Two months after surgery, recurrence of the malignant phyllodes tumor with right axillary lymph node involvement and lung metastasis was confirmed.
\end{abstract}

Conclusion: Initial surgical resection of giant phyllodes tumors is often challenging. For initial presentation with unresectable giant phyllodes tumor, we recommend to perform TACE prior to surgery. In our patient, preoperative TACE was effective and safe. If the tumor has invaded the chest wall, early radiotherapy after surgery may be recommended for preventing recurrence.

Keywords: Malignant phyllodes tumor, Angiosarcoma, Transcatheter arterial chemoembolization, Embozene microspheres

\footnotetext{
* Correspondence: drtu@h.tmu.edu.tw

${ }^{\dagger}$ Chih-Yu Kuo and Shing-Huey Lin contributed equally to this work.

${ }^{3}$ Division of Breast Surgery, Department of Surgery, Taipei Medical University

Hospital, Taipei, Taiwan

${ }^{4}$ Department of Surgery, School of Medicine, College of Medicine, Taipei

Medical University, Taipei, Taiwan

Full list of author information is available at the end of the article
}

(c) The Author(s). 2019 Open Access This article is distributed under the terms of the Creative Commons Attribution 4.0 International License (http://creativecommons.org/licenses/by/4.0/), which permits unrestricted use, distribution, and reproduction in any medium, provided you give appropriate credit to the original author(s) and the source, provide a link to the Creative Commons license, and indicate if changes were made. The Creative Commons Public Domain Dedication waiver (http://creativecommons.org/publicdomain/zero/1.0/) applies to the data made available in this article, unless otherwise stated. 


\section{Background}

Phyllodes tumor of the breast is a rare fibroepithelial lesion composed of both stromal and epithelial components, accounting for $0.3-1.0 \%$ of breast tumors [1]. Some of these tumors may grow rapidly and reach a large size ( $>10 \mathrm{~cm}$ in diameter). After resection of the primary tumor, the reconstruction generally includes skin grafting, which involves harvesting of skin from another body part of the patient [2]. However, skin grafts may cause disease or adverse effects in patients, especially patients with large skin defects or contaminated wounds. Increased size and complexity of chest wall resections could require advanced reconstructive techniques.

TACE is performed by an interventional radiologist to restrict the blood supply of a tumor to cause tumor necrosis and shrinkage. Several studies have shown that preoperative tumor shrinkage has a good prognosis for advanced breast cancer metastasis [2-4]. Here, we report a case of a patient with a giant malignant phyllodes tumor that was successfully reduced through preoperative TACE. In the English literature, only one case has been reported that is similar to our experience. Hashimoto et al. (2016) reported on the preoperative chemoembolization of a large malignant phyllodes tumor with successful avoidance of skin grafting after excision [2].

\section{Case presentation}

A 51-year-old woman without any underlying systemic disease presented with a 1-year history of a bulky tumor in her right breast. She denied having any breast cancer history in her family. The tumor grew rapidly with a large fungating wound and necrotic edges (Fig. 1). She denied having undergone any treatment before the presentation. She was found to have an elevated white blood cell count $\left(11.420 / \mathrm{mm}^{3}\right)$ and a normal tumor marker levels of carcinoembryonic antigen (CEA) and carcinoma antigen 15-3 (CA153). She was hospitalized in our breast medical center and underwent surgical debridement. Antibiotics were administered to control the infection.

Histological examination performed using a core needle biopsy (CNB) showed a spindle cell lesion that was positive for cluster of differentiation 34, hematopoietic progenitor cell antigen (CD34) and negative for beta-catenin, cytokeratin (CK), S100 calcium binding protein (S100), and signal transducer and activator of transcription 6 (STAT6). Because CK staining was negative, metaplastic carcinoma was unlikely. A definite diagnosis could not be established based on CNB specimen findings. Breast sonography revealed a huge heterogeneous mass. Breast CT showed a huge lobulated heterogeneously enhancing mass, measuring approximately $19.43 \times 12.98 \times 21.47 \mathrm{~cm}$, in the right breast (Fig. $2 \mathrm{a}$ and b). No evidence of distant metastasis was observed with whole-body CT. A bone scan revealed no evidence of skeletal metastasis. Magnetic resonance imaging (MRI) revealed major pectoralis muscle invasion and several enlarged lymph nodes in the right axillary region (Fig. 2c and d).

Considering that the patient's breast tumor is inoperable, because the entire chest wall tissue diffuse necrosis and skin defect after mastectomy may over $30 \times 25 \mathrm{~cm}$, we performed preoperative intra-arterial infusion chemotherapy and embolization to shrink the tumor before mastectomy. Four cycles of $30 \mathrm{mg}$ of epirubicin plus embozene microspheres were administered with a 1-week interval between cycles (Table 1).

For chemoembolization, after the administration of local anesthesia, a angiosheath was inserted into the right femoral artery, followed by the introduction of an angiocatheter and a microcatheter. The vascular blood supplies of the tumor, right internal mammary artery,
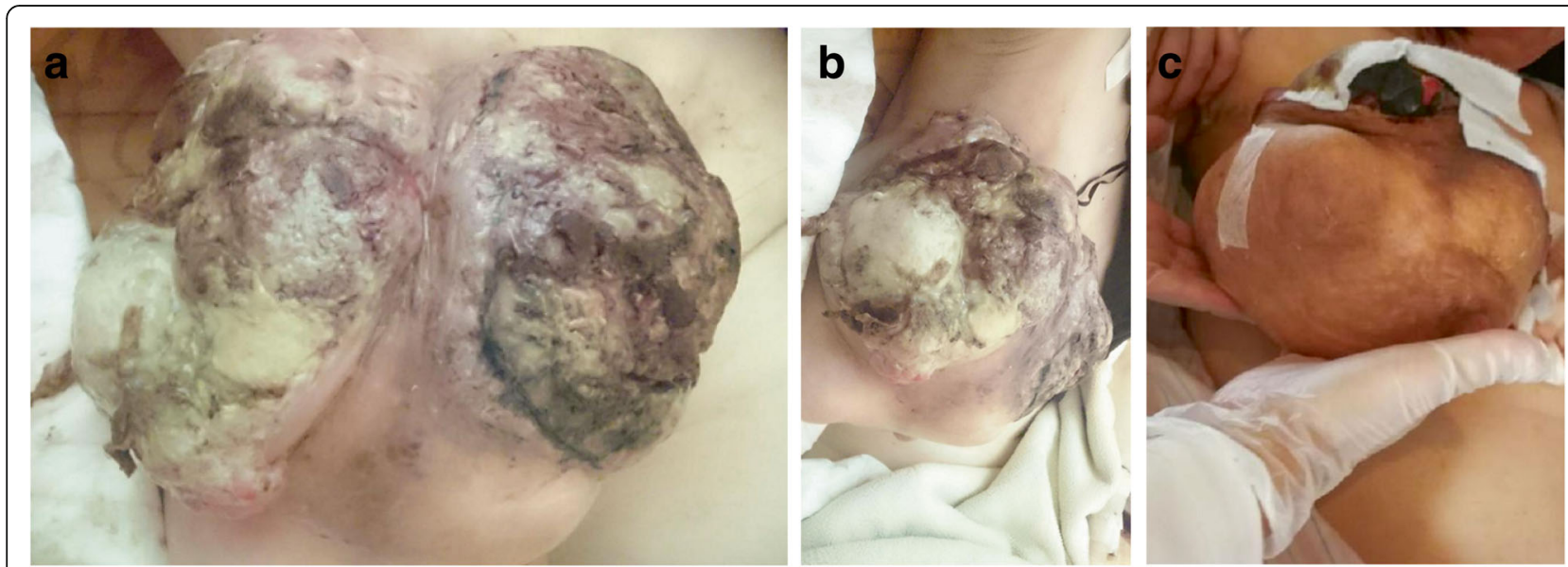

Fig. 1 a Gross tumor before treatment. Entire breast surface revealed a fungating wound and necrotic changes. $\mathbf{b}$ and $\mathbf{c}$ Shrinkage of the gross tumor observed after four cycles of preoperative chemoembolization 

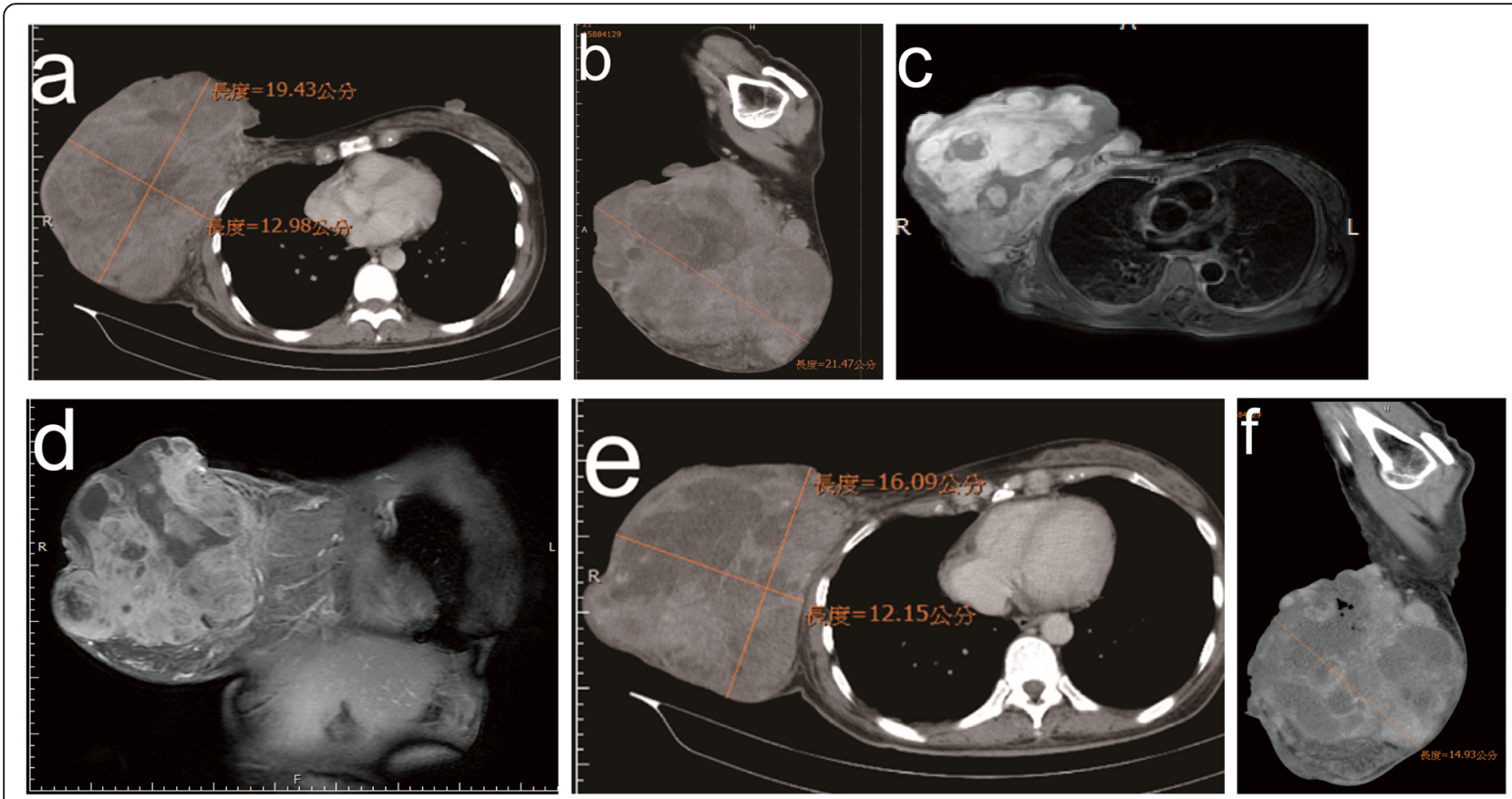

Fig. $\mathbf{2}$ a and $\mathbf{b}$ Contrast-enhanced chest CT showed a huge lobulated heterogeneously enhancing mass (measuring approximately $19.43 \times$ $12.98 \times 21.47 \mathrm{~cm}$ ) in the right breast. $\mathbf{c}$ and $\mathbf{d}$ Contrast-enhanced MRI showed a 20-cm lobulated right breast mass with right major pectoralis muscle invasion. e and $\mathbf{f}$. After four courses of TACE, follow-up CT revealed tumor reduction of $45 \%$ of total volume (measuring approximately $16.0 \times 12.0 \times 15.0 \mathrm{~cm})$

thoracoacromial artery, lateral thoracic artery, and thoracodorsal artery were identified (Fig. 3). A pulsed injection of $30 \mathrm{mg}$ of epirubicin was administered into the tumor through the microcatheter. Subsequently, embozene microspheres were injected.

After chemoembolization, fever and right chest pain at the tumor site were noted. Symptoms resolved within 2 days. No other serious complications were observed after TACE. Follow-up CT revealed a $45 \%$ reduction in total tumor volume (Fig. 2e and f). Next, simple mastectomy was performed. Skin grafting was not performed during surgery (Fig. 4). The tumor-free margin was reported after further pathological examination.

The right breast measured $26 \times 20 \times 10 \mathrm{~cm}$ in size and $1835 \mathrm{~g}$ in weight. Pathological findings indicated the presence of a malignant phyllodes tumor with an angiosarcoma component. The surgical margin was not involved by the tumor. In addition, the nipple and axillary nodes were not involved by the tumor.

The surgery successfully treated tumor pain and caused tumor necrosis. Because the tumor invaded the chest wall muscle layer, we speculated that concerning microscopic

Table 1 Diameter of Embosphere Microspheres, Epirubicin used and the embolization vascular in each procedure

\begin{tabular}{llll}
\hline & The diameter of Embosphere Microspheres (micron) & Epirubicin $(\mathrm{mg})$ & The embolization vascular \\
\hline First procedure & 400,500 & 30 & Right internal mammary artery \\
Second procedure & 400 & 30 & Thoracoacromial artery \\
& & Lateral thoracic artery & Thoracodorsal artery \\
Third procedure & $400,500,700$ & 30 & Right internal mammary artery \\
& & Lateral thoracic artery & Thoracodorsal artery \\
Forth procedure & 400,700 & 0 & Right internal mammary artery \\
& & Thoracodorsal artery &
\end{tabular}



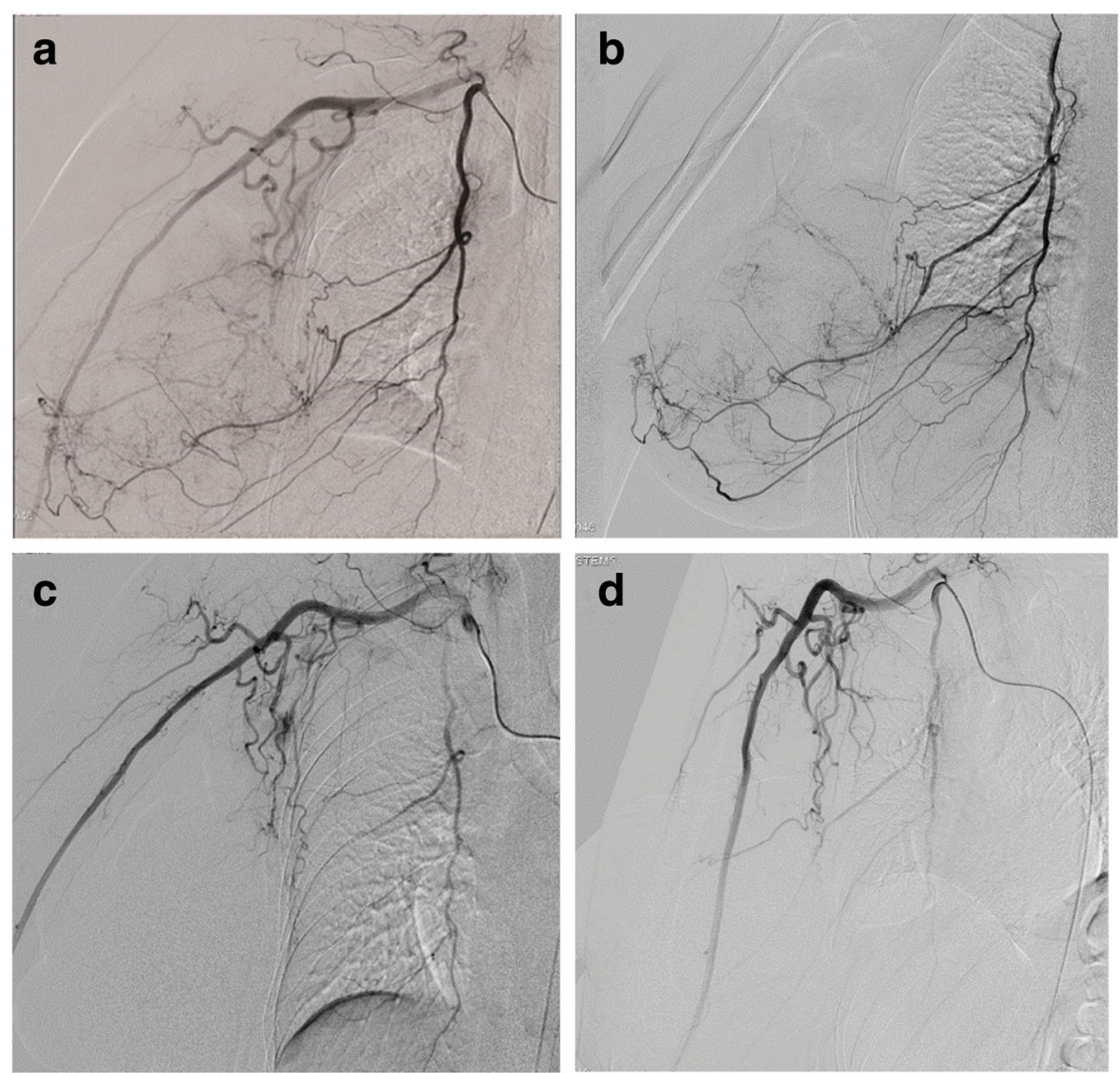

Fig. 3 Arteriography identified the vascular blood supply of the tumor. a Right subclavian artery. b Internal mammary artery. c Lateral thoracic and thoracodorsal arteries. $\mathbf{d}$ Post-TACE showed all target tumor blood supply vessels

foci could have remained. Therefore, we recommended the patient undergo adjuvant irradiation to prevent recurrence. However, the patient rejected this therapeutic option and was lost to follow-up for 2 months.

When the patient presented again 2 months after surgery, we noted the development of a palpable mass over the right chest wall. A palpable lymph node was observed in the right axillary region. Follow-up CT showed a mass measuring $5.0 \times 4.0 \mathrm{~cm}$ over the right chest wall and a palpable lymph node measuring $6.0 \times 5.0 \mathrm{~cm}$ was found in the right axillary region, beneath the pectoralis muscle. Additionally, several ground glass lesions were
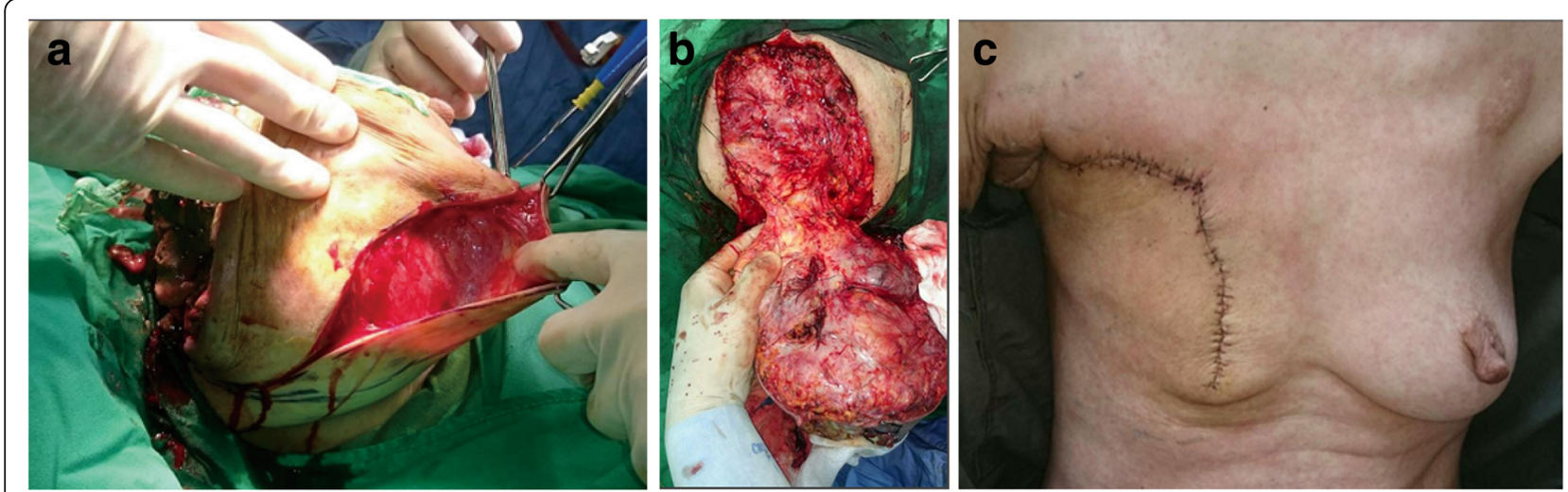

Fig. 4 a and $\mathbf{b}$ Simple mastectomy was performed. c After surgery, the tumor was completely resected without skin grafting 
noted in the bilateral lungs. CNB findings confirmed lymph node metastasis. Therefore, malignant phyllodes tumor recurrence with axillary lymph node involvement and lung metastasis was diagnosed. Furthermore, pathology and immunohistochemistry (IHC) findings confirmed the presence of a malignant phyllodes tumor with recurrence. The patient obtained four courses of additional chemotherapy and two fractions of radiotherapy after recurrence was found ( 2 months after surgery). After that, she refused further treatment and was lost to follow-up.

\section{Discussion and conclusions}

CNB is widely used as a highly sensitive method for obtaining a preoperative diagnosis of breast cancer [5]. However, diagnostic challenges in pathological interpretation and controversies regarding the management of certain lesions diagnosed through percutaneous CNB remain. Although histological features, IHC findings, and genetic test results can help establish a diagnosis, the overlap of pathological features and small amount of the tissue obtained in CNB cause difficulty in the diagnostic classification of these lesions [6].

In our case, the pathological findings of the first $\mathrm{CNB}$ specimen revealed spindle tumor cell proliferation with mild atypia on the dense collagenous stromal background. Diagnosis of a spindle cell lesion in the breast is particularly difficult when encountered in CNB findings. Several diagnoses should be considered for a spindle cell lesion of the breast. Because of our patient's clinical appearance, the presence of a malignant lesion was highly suspected, including a malignant phyllodes tumor, metaplastic carcinoma, melanoma, angiosarcoma, or other primary breast sarcoma [7].

Distinguishing metaplastic carcinoma and malignant phyllodes tumors of the breast is critical because their treatment and prognosis differ significantly. The leaf-like architecture and absence of CK expression can be helpful markers of phyllodes tumor [8]. The pathological findings of our case after surgery showed a leaf-like structure (Fig. 6a). The stromal component revealed increased cellularity, moderate nuclear pleomorphism, increased mitotic activity, and stromal overgrowth (Fig. 6b). All these pathological features are often observed in malignant phyllodes tumors. IHC is a useful tool in the diagnosis of a spindle cell lesion. In our case, IHC results were positive for CD34 but negative for beta-catenin, CK (AE1/AE3), S-100, and STAT6. Although no marker is perfectly sensitive or specific, the majority of phyllodes tumors are positive for CD34, and CD34 expression is seldom seen in spindle cell metaplastic carcinoma. Anastomosing and dissecting vascular channels lined by flat atypical cells in the vascular space were observed (Fig. $5 \mathrm{c}$ and d). Pleomorphic cells are positive for cluster of differentiation 31, platelet endothelial cell adhesion molecule (CD31) (Fig. 6e) and erythroblast transformation-specific related gene (ERG) but negative for CK (Fig. 6f). Therefore, IHC performed to distinguish carcinoma from angiosarcoma should include both epithelial and endothelial markers $[9,10]$. Based on the histological features and results of $\mathrm{IHC}$, our patient was diagnosed with a malignant phyllodes tumor of the breast with an angiosarcoma component.

Occasionally, specific heterologous sarcomatous elements, including liposarcoma, osteosarcoma, chondrosarcoma, fibrosarcoma, and rhabdomyosarcoma, may be observed in phyllodes tumors [11]. Such malignant phyllodes tumors account for approximately $20 \%$ of all cases. The heterologous angiosarcoma component is considerably rare, and only four cases of angiosarcoma developing in the clinical setting of a phyllodes tumor have been reported [12-15]. Our case is the fifth case in the literature.

When the chest wall defect is over $300 \mathrm{~cm}^{2}$, free tissue transfer or multiple flaps are warranted [16]. Sometimes, prosthetic mesh should be used at the defect or weak part for reinforcement. Most cases are reconstructed successfully, but some have complications, such as flap loss, haematoma, infection, delayed wound healing,
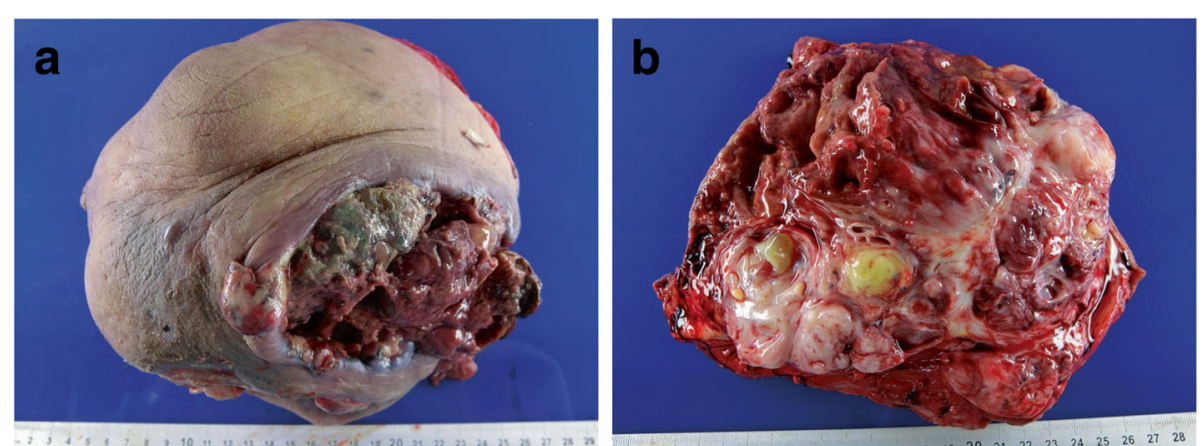

Fig. 5 a The right breast measured $26.0 \times 20.0 \times 10.0 \mathrm{~cm}$. The skin was covering it elliptical in shape with ulcerative changes. b Anastomosing and dissecting vascular channels were found 

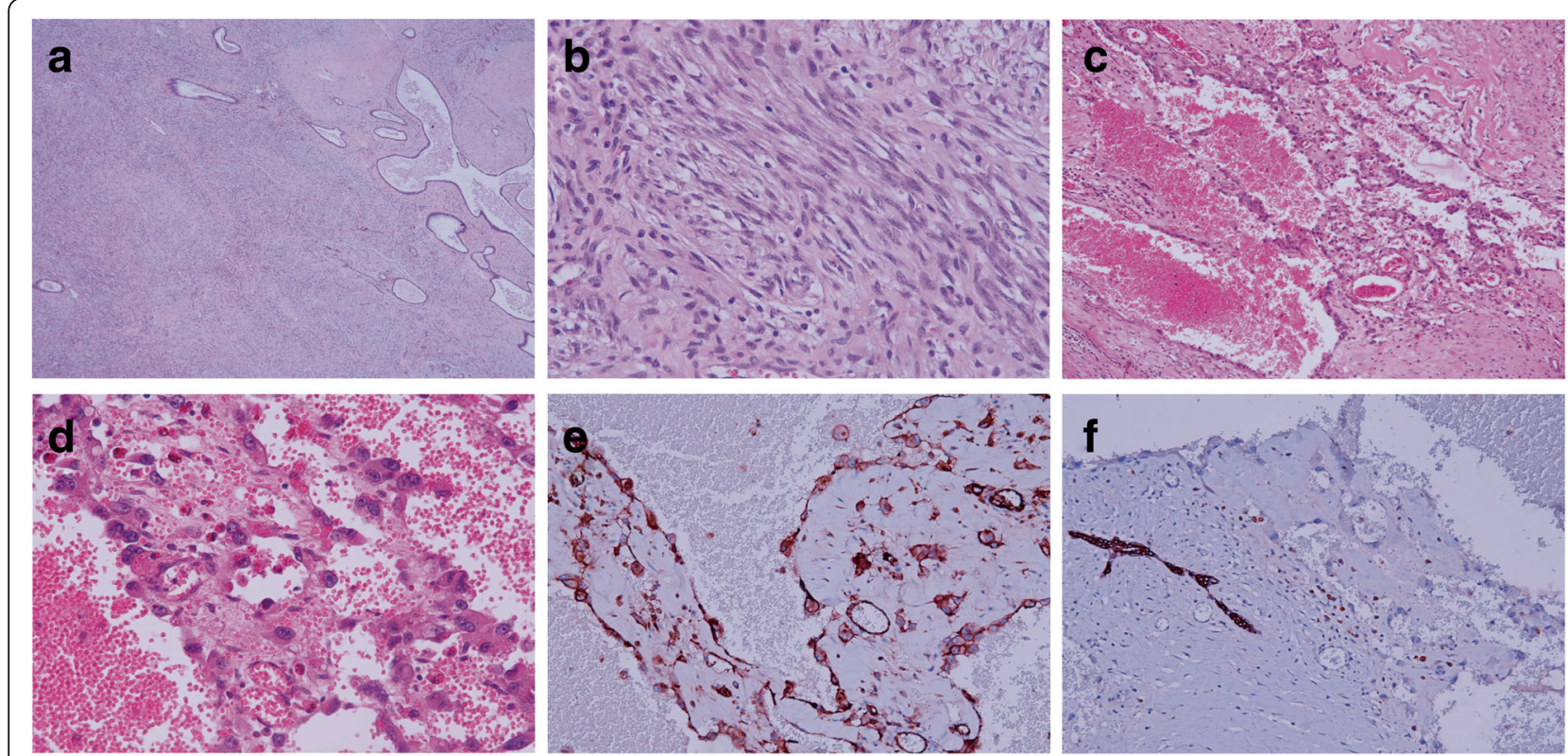

Fig. $\mathbf{6}$ a Stromal component showed leaf-like appearance. b Stromal overgrowth with a spindle cell feature. $\mathbf{c}$ and $\mathbf{d}$ Atypical cells in the vascular space. e Vascular endothelium present with positive CD31. f CK negative in the vascular space, CK staining positive at the ductal epithelium

dehiscence or vascular thrombosis. However, cases with chest wall defect over $30 \times 25 \mathrm{~cm}$ are rare. Joo Seok Park et al. [17] and Romain Bosc et al. [18] reported that only one case had chest wall defect over $700 \mathrm{~cm}^{2}$ in their studies. Increased size and complexity of chest wall resections could require advanced reconstructive techniques. The technique is still challenging and needs more experience.

TACE is used in locally advanced breast cancer for downstaging and increasing resectability [19]. TACE with superabsorbent polymer microspheres is a well-tolerated and feasible palliative option for patients with pulmonary or mediastinal metastasis from breast cancer [20]. For giant phyllodes tumors, only one case report demonstrated the effect of preoperative chemoembolization. Our case of a giant malignant phyllodes tumor is the second to undergo simple mastectomy without skin grafting through preoperative chemoembolization, which was useful in reducing tumor size. Treatment procedures were successful with no Common Terminology Criteria for Adverse Events (CTCAE) grade 3/4 toxicity observed. Laboratory data revealed no thrombocytopenia, hyperbilirubinemia, hypoleucocytosis, or failure of hepatic or renal functions. TACE causes postembolization syndrome, with localized pain, fever, vomiting, nausea, and fatigue being the commonest adverse events [21]. In our case, only fever and localized pain were noted after several days of TACE. Therefore, we confirm that TACE was highly effective in shrinking the giant phyllodes tumor in our patient and is a safe procedure.
In our study, the tumor had invaded the right pectoralis major. Chest wall invasion is an uncommon event. Reinfuss et al. reported 170 women with phyllodes tumors, $2.4 \%$ of cases with pectoralis major invasion [22]. Such cases were treated through radical resection with chest wall reconstruction [23]. Because breast CT after TACE indicated shrinkage of the right breast tumor, our patient did not undergo chest wall resection and reconstruction. However, tumor recurrence was discovered over the right chest wall 1 month after surgery. According to the literature, local recurrence rates after margin-negative breastconserving resections of malignant phyllodes tumours are unacceptably high, at 23 to 30\% [24]. Margin-negative resection combined with adjuvant radiotherapy is a very effective therapy for local control of malignant phyllodes tumours [25]. Therefore, early radiotherapy after surgery may be recommended for preventing recurrence.

In summary, initial surgical resection of giant phyllodes tumors is challenging. We recommend performing TACE prior to surgery. Preoperative TACE was effective and safe for our patient. If the tumor has invaded the chest wall, early radiotherapy after surgery may be recommended for preventing recurrence. Additional studies are required to establish and demonstrate the efficacy of this procedure.

\section{Abbreviations}

CA153: Carcinoma Antigen 15-3; CD31: Cluster of differentiation 31, platelet endothelial cell adhesion molecule; CD34: Cluster of differentiation 34, hematopoietic progenitor cell antigen; CEA: Carcinoembryonic antigen; CK: Cytokeratin; CNB: Core needle biopsy; CT: Computed tomography; 
CTCAE: Common Terminology Criteria for Adverse Events; ERG: Erythroblast transformation-specific related gene; IHC: Immunohistochemistry; MRI: Magnetic resonance imaging; S100: S100 calcium binding protein; STAT6: Signal transducer and activator of transcription 6; TACE: Transcatheter arterial chemoembolization

\section{Acknowledgements}

Not applicable.

\section{Authors' contributions}

CYK and SHL collected, analysed and interpreted the data and drafted the manuscript. SJC performanced the TACE procedure. JSC examined the pathologic pictures of the tumor. SHT, KDL designed and supervised the study, interpreted the data, and critically revised the manuscript. All authors have read and approved the final version of this manuscript.

\section{Funding}

This study was supported by the Ministry of Science and Technology, Taiwan awarded to Dr. Tu, MOST106-2314-B-038-053-MY3.

\section{Availability of data and materials}

The datasets used and/or analyzed during the current study are available from the corresponding author on reasonable request.

\section{Ethics approval and consent to participate}

This case report was approved and supervised in terms of ethics, consent, and patient confidentiality by the Institutional Review Board of Taipei Medical University, Taipei, Taiwan (TMUJIRB, N201810001). The written informed consent was obtained from the patient's daughter to participate to this case report and any accompanying images.

\section{Consent for publication}

A written informed consent for publication of the case's clinical information and clinical images was obtained from the patient's daughter. A copy of the consent for publication is available for review by the editor of this journal.

\section{Competing interests}

The authors declare that they have no competing interests.

\section{Author details}

${ }^{1}$ Department of Surgery, Taipei Medical University Hospital, Taipei, Taiwan. ${ }^{2}$ Division of Family Medicine, Cathay General Hospital, Taipei, Taiwan. ${ }^{3}$ Division of Breast Surgery, Department of Surgery, Taipei Medical University Hospital, Taipei, Taiwan. ${ }^{4}$ Department of Surgery, School of Medicine, College of Medicine, Taipei Medical University, Taipei, Taiwan. ${ }^{5}$ Taipei Cancer Center, Taipei Medical University, Taipei, Taiwan. ${ }^{6}$ Division of Hematology and Oncology, Department of Internal Medicine, Taipei Medical University Hospital, and School of Medicine, College of Medicine, Taipei Medical University, Taipei, Taiwan. ${ }^{7}$ Department of Medical Imaging, Taipei Medical University Hospital, Taipei, Taiwan. ${ }^{8}$ Department of Pathology, School of Medicine, College of Medicine, Taipei Medical University, Taipei, Taiwan.

\section{Received: 15 April 2019 Accepted: 15 July 2019}

Published online: 27 July 2019

\section{References}

1. Zhang Y, Kleer CG. Phyllodes tumor of the breast: histopathologic features, differential diagnosis, and molecular/genetic updates. Arch Pathol Lab Med. 2016;140(7):665-71.

2. Hashimoto K, Mimura H, Arai Y, Doi M, Kojima Y, Tsugawa K, et al. Successful preoperative chemoembolization in the treatment of a Giant malignant Phyllodes tumor. Cardiovasc Intervent Radiol. 2016;39(7):1070-5.

3. Tozaki M, Kobayashi T, Uno S, Aiba K, Takeyama H, Shioya H, et al. Breastconserving surgery after chemotherapy: value of MDCT for determining tumor distribution and shrinkage pattern. AJR Am J Roentgenol. 2006;186(2):431-9.

4. Expert Panel on Breast I, Slanetz PJ, Moy L, Baron P, di Florio RM, Green ED, et al. ACR appropriateness criteria((R)) monitoring response to neoadjuvant systemic therapy for breast cancer. J Am Coll Radiol 2017;14(11S):S462-SS75.

5. Dillon MF, Quinn CM, McDermott EW, O'Doherty A, O'Higgins N, Hill AD. Needle core biopsy in the diagnosis of phyllodes neoplasm. Surgery. 2006;140(5):779-84.
6. Corben $A D$, Edelweiss $M$, Brogi $E$. Challenges in the interpretation of breast core biopsies. Breast J. 2010;16(Suppl 1):S5-9.

7. Rakha EA, Aleskandarany MA, Lee AH, Ellis IO. An approach to the diagnosis of spindle cell lesions of the breast. Histopathology. 2016;68(1):33-44.

8. Rungta S, Kleer CG. Metaplastic carcinomas of the breast: diagnostic challenges and new translational insights. Arch Pathol Lab Med. 2012;136(8):896-900.

9. Miettinen M, Wang ZF, Paetau A, Tan SH, Dobi A, Srivastava S, et al. ERG transcription factor as an immunohistochemical marker for vascular endothelial tumors and prostatic carcinoma. Am J Surg Pathol. 2011;35(3):432-41.

10. Miettinen $M$, Lindenmayer AE, Chaubal A. Endothelial cell markers CD31, $\mathrm{CD} 34$, and $\mathrm{BNH} 9$ antibody to $\mathrm{H}$ - and $\mathrm{Y}$-antigens--evaluation of their specificity and sensitivity in the diagnosis of vascular tumors and comparison with von Willebrand factor. Mod Pathol. 1994;7(1):82-90.

11. Tan BY, Acs G, Apple SK, Badve S, Bleiweiss IJ, Brogi E, et al. Phyllodes tumours of the breast: a consensus review. Histopathology. 2016;68(1):5-21.

12. Tranesh G, Nassar A. Malignant phyllodes tumor of the breast with heterologous high-grade angiosarcoma. Human Pathol Case Rep. 2017;7:57-61.

13. Costa S, Graca SA, Ferreira A, Maciel J. Breast angiosarcoma secondary to phyllodes tumour. BMJ Case Rep. 2012;2012:bcr2012007545.

14. Kumar M, Goel MM, Sagar M, Tiwari S. Angiosarcoma in recurrent malignant phyllodes tumor of breast. Indian J Pathol Microbiol. 2012;55(2):264-5.

15. Mamoon N, Mushtaq S, Hassan U, Rashid R, Khadim MT, Sarfraz T, et al. Angiosarcoma arising in recurrent phyllodes tumour. Histopathology. 2009;54(7):913-6.

16. Chang RR, Mehrara BJ, Hu QY, Disa JJ, Cordeiro PG. Reconstruction of complex oncologic chest wall defects: a 10-year experience. Ann Plast Surg. 2004;52(5):471-9 discussion 9.

17. Park JS, Ahn SH, Son BH, Kim EK. Using local flaps in a chest wall reconstruction after mastectomy for locally advanced breast cancer. Arch Plast Surg. 2015;42(3):288-94.

18. Bosc R, Lepage C, Hamou C, Matar N, Benjoar MD, Hivelin M, et al. Management of chest wall reconstruction after resection for cancer: a retrospective study of 22 consecutive patients. Ann Plast Surg. 2011;67(3):263-8.

19. Kitagawa K, Yamakado K, Nakatsuka A, Tanaka N, Matsumura K, Takeda K, et al. Preoperative transcatheter arterial infusion chemotherapy for locally advanced breast cancer (stage IIlb) for down-staging and increase of resectability. Eur J Radiol. 2002;43(1):31-6.

20. Kennoki N, Hori S, Yuki T, Hori A. Transcatheter arterial chemoembolization with spherical embolic agent in patients with pulmonary or mediastinal metastases from breast Cancer. J Vasc Interv Radiol. 2017;28(10):1386-94.

21. Ni JY, Sun HL, Chen YT, Luo JH, Wang WD, Jiang XY, et al. Drug-eluting bead transarterial chemoembolization in the treatment for unresectable soft tissue sarcoma refractory to systemic chemotherapy: a preliminary evaluation of efficacy and safety. J Cancer Res Clin Oncol. 2018;144(1):157-63.

22. Reinfuss M, Mitus J, Duda K, Stelmach A, Rys J, Smolak K. The treatment and prognosis of patients with phyllodes tumor of the breast: an analysis of 170 cases. Cancer. 1996;77(5):910-6.

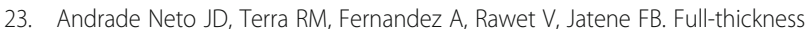
chest wall resection for recurrent breast phyllodes tumor. Ann Thorac Surg. 2007:83(6):2196-7.

24. Lu Y, Chen Y, Zhu L, Cartwright P, Song E, Jacobs L, et al. Local recurrence of benign, borderline, and malignant Phyllodes tumors of the breast: a systematic review and meta-analysis. Ann Surg Oncol. 2019;26(5):1263-75.

25. Barth RJ Jr, Wells WA, Mitchell SE, Cole BF. A prospective, multi-institutional study of adjuvant radiotherapy after resection of malignant phyllodes tumors. Ann Surg Oncol. 2009;16(8):2288-94.

\section{Publisher's Note}

Springer Nature remains neutral with regard to jurisdictional claims in published maps and institutional affiliations. 